\title{
Analyse linguistique du droit applicable aux demandeurs d'asile: l'exemple de Mariama
}

\author{
Anne Triboulet
}

\section{Précis}

A travers l'étude comparative de deux décisions jurdictionnelles, cet article présente une analyse linguistique du droit applicable, en France, aux demandeurs d'asile. D'une part, l'auteur souligne l'importance du style littéraire utilisé dans la rédaction des jugements et démontre le rôle du «storytelling ", la façon dont est présentée le demandeur d'asile, dans la décision prise quant au fond. $D$ 'autre part, elle analysele «langagedu droit afin de mettre en mettre en reliefla façon dont la structure de la pensée juridique s'écarte de la logique formelle et $d u$ bon sens et permet ainsi de justifier, juridiquement, des décisions prises al'égard de demandeurs d'asile qui ne peuvent se justifier rationnellement.

\section{Abstract \\ Through a comparative study of two court decisions, this article presents a lin- guistic analysis of the applicable rules to asylum applicants in France. First, the author underlines the importance of the literary style in the drafting of decisions on the merits. Second, she analyzes the "language of the law" to show how the structure of legal thought sometimes dif- fers from formal logic and common sense, thus justifying legal decisions taken with regard to asylum applicants that could not be rationally justified.}

Cet article a pour objectif de souligner l'importance du langage dans le droit applicable, en France, aux demandeurs

Anne Triboulet, Mattrisede Droit Public, LL.M, est actuellement stagiaire au Sizge des Nations Unies d New York, au sein du Bureau de lo Coordination des Affaires humanitaires (Groupe de developpement des politiques).

L'auteur remercie le professeur Marie-Claude Premont de l'Université McGill, qui a été à l'origine de cet article (Voir M-C Premont, Le Langage du Droit, These de Doctorat, Universite Laval, Janvier 1996). d'asile. En effet, lors del'examen de cas individuels, de nombreux facteurs extra-juridiquesjouent un rôle important. Lelangage estl'und'entreeuxetlechoix des mots employésn'est jamais neutre. Dans le même temps, puisqu'il existe un langage du droit, l'analyse linguistique est un outil privilégié pour étudier la structure de la pensée juridique. Nous privilégierons pour cela l'analyse des tropes. Cette tournure de langage est, en effet, intéressante car les mots y prennent une signification différente deleur signification propre. Or, la logiquejuridique est fondée sur un détournement dela signification propre des mots, pour leur donner une significationjuridique. Parmi les tropes analysés, nous accorderons une place privilégiée à la recherche des métaphores ${ }^{1}$ et des métonymies ${ }^{2}$, ainsi qu'aux raisonnements métaphoriques et métonymiques. Il s'agira de s'interroger sur la signification de leur emploi, surce qu'ils servent à mettre en lumière, et donc, ce qu'ils laissent dans l'ombre.

L'étude d'un cas particulier semble la manière la plus adéquate de mettreen évidencele rôle joué par le langage dans les procédures juridiques applicables aux demandeurs d'asile. Ainsi, nous présenterons tout d'abord l'histoire de Mariama et les deux décisions qui ont été prises à sonégard. Puis, nousétudierons la qualification et l'interprétation des faits par les deux instances juridictionnelles et, leurs raisonnements juridiques. Enfin, nous nous intéresserons aux conséquences de ces décisions.

\section{L'histoire juridique de Mariama}

Mariama est de nationalité guinéenne. Elle appartient à l'ethnie et à l'entourage de l'ancien président Sékou TOURE. A la mort dece dernier, en 1984, la famille de Mariama a été victime de vengeances populaires. Mariama est alors allée en France où elle a demandé l'asile politique mais a êté déboutée et reconduite en Guinée. De retour dans son village, la population, la reconnaissant, a tenté dela lyncher. Elles'est alors réfugiée chez son oncle à Conakry mais a dû s'enfuir de nouveau car ce dernier projetait d'exciser sa fille. Ainsi, en 1995, Mariama a déposé une nouvelle demande de reconnaissance de la qualité de réfugiée en France basée sur ses craintes de persécution en raison de ses origines ethniques et familiales. Elle soutenait également que ses filles risquaient d'être persécutées en raison de leur appartenance «au groupe social des femmes qui sont victimes de mutilations sexuelles et de pratique contraires à la dignité humaine, volontairement tolérées, au nom de la tradition, par les autorités publiques de son pays d'origine" 4 . Se prononçant conformément à la Convention de $1951^{5}$ et du Protocole de New York de 1967, l'Office Français pour la Protection des Réfugiés et des Apatrides (ciaprès OFPRA), puis par la Commission des Recours des Réfugiés (ci-après CRR) rejetteront sa demande, $n$ 'estimant pas fondées ses craintes de persécutions. ${ }^{6}$

Parallèlement à cette première procédure, Mariama a fait l'objet d'une ordonnance d'interdiction du territoire prononcée parle tribunal correctionnel de Saint-Etienne pour avoir utilisé et tenté d'obtenir de faux documents administratifs. En exécution de cette ordonnance, le préfet de Loire a pris à son encontre un arrêté de reconduite à la frontière vers la Guinée. Le tribunal administratif de Lyon ${ }^{7}$, suivant les conclusions du Commissaire du Gouvernement, a annulé cet arrêté au motif que Mariama «ne serait pas en mesure de s'opposer à la volonté de sa belle-famille de procéder à l'excision de ses deux fillettes». Or, l'excision est un traitement contraire à l'article 3 de la Convention européenne de sauvegarde des droits de l'homme et des libertés fondamentales ${ }^{8}$ et l'article 27 bis de l'ordonnance du 2 novembre 1945 interdit d'éloigner une personne à destina- 
tion d'un pays où elle est exposée à des traitements contraires à l'article 3 précité.

\section{Une qualification et une interprétation des faits au service de raisonnements métonymiques}

Durant l'entre deux guerres, l'auteur A. L. Goodhart insistait déjà sur le fait que

facts are not constant but relative. [...] The same set of facts may look entirely different to two different persons. The judge founds his conclusions upon a group of facts selected by him as material, from among a larger mass of facts [...]. The judge, therefore, reaches a conclusion upon de facts as he sees them. It is on these facts that he bases his judgement, and not on any other. [...] A congeries of facts is presented to him ; he chooses those which he considers material and rejects those which are immaterial, and then bases his conclusions on the material ones. To ignore his choice is to miss the whole point of the case'.

Tel est effectivement le processus par lequel la CRR et le tribunal administratif sont parvenus, à partir des mêmes faits, à des conclusions différentes.

Le principal raisonnement métonymique utilisé consiste à avoir statué sur l'ensemble du cas en ne se basant que sur certains éléments des faits présentés. En considérant «une partie pour le tout", ceprocédé laissenécessairement dans l'ombre une autre partie de la demande, tout aussi importante et qui aurait conduit à une autre conclusion si elle avaitétéégalement prise en compte.

Ainsi, bien que le Conseil d'Etat ait reconnu qu'en vertu du principe général de l'unité de la famille, un demandeur $d$ 'asile peut invoquer les risques de persécutions encourus par ses enfants ${ }^{10}$, la décision de la CRR ne s'attarde que sur les faits qui concernent personnellementMariama, et nonsurla situation de ses filles. La technique utilisée par laCRR pour appuyer son choix est celle du «storytelling». En effet, La CRRprésente Mariama d'une façon qui ne lui est pas favorable, insistant sur son lien de parenté avec l'ancien présidentSékou TOURE et précisant qu'elle et sa famille ont «joui» des faveurs de son régime. Cette présentation conduit à la considérer comme «complice» du régime dictatorial de Sékou TOURE et suggère une certaine légitimité dans les agissements de la population guinéenneà sonégard. Ainsi la CRR considérera «qu'iln'est pasétabliqueles membres del'ethnie Malinképroches del'ancien président Sékou TOURE continueraientd'être victimes de menaces volontairement tolérées par les autorités publiques", sans qu'elleaitàmotiver cette affirmation car ellesemble découler naturellementdela présentation des faits ${ }^{11}$.

A l'inverse, la décision du tribunal administratif de Lyon offre une image beaucoup plus positive de Mariama, laissant de côté l'aspect politique de sa demaride et la présentant comme une mère de famille qui cherche à protéger ses enfants des risques d'excision qu'elles encourent. Denouveau, la technique du "storytelling" est utilisée. Ainsi, le tribunal n'emploie pas les termes neutres de "filles" ou "enfants" de Mariama mais celui de "fillettes", plus attendrissant. Il renforce également le sentiment de proximité en personnalisant les «fillettes» en citant leurs prénoms, Sakahlé et Diankenba.

Le deuxième raisonnement métonymique auquel se sont livrés la $C R R$ et le tribunal tient à l'interprétation de la condamnation de Mariama pour avoir fait usage de faux documents administratifs. En effet, en 1994, Mariama et son époux avaient tenté de faire établir la nationalité française de leursenfants en démontrant que leur père était français car né dans une colonie française. Or l'époux de Mariama était né après l'indépendance de la Guinée. La CRR, bien que reconnaissant que Mariama ne savaitnilireniécrire et qu'elleétait victime de violences conjugales, met l'accent sur la conséquence de son acte, qualifié très négativement de tentative "par des moyens frauduleux d'obtenir ind ument la délivrance de documents administratifs». En revanche, le Commissaire du Gouvernement insiste sur les causes des agissements de Mariama. Il meten effet en relief sa volonté de rester en France, qualifiant la fraude de sforme particulièred'attachement à la France». II justifie égalementl'action de Mariama en insistant sur les nombreuses difficultés administratives et judiciaires qu'elle a rencontrées. Il insiste sur l'aspect laborieux des procédures et emploie le mot " refus " trois fois dans le même paragraphe de façon à traduire l'hostilité des autorités françaises (le refus de l'OFPRA, de la CRR et du préfet) qui a conduit Mariama à effectuer de nombreuses démarches «en vain». L'accent ainsi porté sur les causes des agissements deMariama conduità en atténuer les conséquences, à passer sous silence le caractère frauduleux de tels actes afin de les qualifier de tentative «de faireétablir la nationalité française de leurs enfants".

\section{Le raisonnement de la Commission des Recours des Réfugiés et du tribunal administratif}

Les décisions quant au fond prises par les deux instances découlent directement de la façon dont les faits ont été présentés. Ainsi, la décision de la CRR se concentre principalement sur l'évaluation des craintes de persécutions de Mariama, alors que celle du tribunal administratif étudie exclusivement les risques d'excision encourus par les enfants de celle-ci.

La CRR a estimé que Mariama ne possédait pas de craintes fondées de persécutions. En effet, en demandantle renouvellement de son passeport, Mariama abénéficiéde la protection de son pays etne peut donc plus prétendre à une protection internationale ${ }^{12}$. De plus, laCRRa estiméque les menaces de la part de la population et volontairement tolérées par les autorités publiques à l'encontre des proches de l'ancien présidentSékou TOURE et desmembres du groupe ethnique Malinké avaient cessés ${ }^{13}$. Cette décision reflèteles principaux mécanismes métaphoriques et métonymiques généralement utilisés dans le cadre de l'examen des demandes d'asile. L'une des étapes nécessaires est la détermination des agents de persécutions, c'est-à-dire, la recherche des "sources" de persécutions. Cette métaphore évoque l'idée de transparence (il existe des "sources" identi- 
fiées) et de garanties contre l'arbitraire (la décision possède un fondementetne tient pas au bon vouloir du juge). L'aspectnégatif de la procédure quela métaphore tend à laisser dans l'ombre est le raisonnement métonymique qui l'accompagne. En effet, identifier les sources de persécutions conduit à devoir en reconnaître certaines et en exclure d'autres. En France, sont généralement reconnues les persécutions qui sont le faits des autorités d'un pays ou le fait de groupes dont les actes sont volontairement tolérées par les autorités publiques ou pour lesquels ces autorités sont incapables d'offrir une protection. Il s'agit d'un raisonnement métonymique dans la mesure où une partie (les persécutions exercées par certains agents) est considérée comme le tout (l'ensemble des persécutions qu'un individu peut craindre et qui peuvent le conduire à fuir son pays). Or, dans de nombreux pays d'Afrique ayant connu un changement brutal de régime politique, les vengeances populaires à l'encontre des partisans du gouvernement précédent durent de nombreuses années, alors même que ces pays sont reconnus en voie de stabilisation par la communauté internationale. Le fait que Mariama ait pris contact avec son ambassade en France ne signifie donc pas, en soi, que l'Etat guinéen ait été en mesure de la protéger dans le pays des lynchages de la population, qui peuvent aboutir à de véritables persécutions.

D'autre part, la CRR a conclue queles craintes de Mariama de voir ses deux filles excisées en cas de retour en Guinée n'étaient pas fondées et «que la position favorable à une telle mutilation qu'aurait antérieurement adoptée un oncle de l'intéressée n'est pas suffisante pour infirmer cette analyse». Il est vrai que dans la société et la culture française, l'attitude et la volonté "d'un oncle", ne sont pas déterminantes. La rédaction tend donc a minimiser les risques d'excision ${ }^{14}$. Elle ne prend pas en compte la conception africaine traditionnelle de la famille au sein de laquellel'autorité d'un oncle peut véritablement s'imposer, et ce d'autant plus que, dans le cas présent, cet oncle constituait la seule famille chez qui Mariama avait pu trouver refuge ${ }^{15}$.

Les conclusions du Commissaire du Gouvernement et la décision du tribunal administratif ont, en revanche, été beaucoup plus favorables à Mariama. Qualifiant l'excision de traitement inhumain et dégradant, elles ont ainsi empêché que Mariama et ses filles soient reconduites en Guinée. Le langage utilisé a été spécialement étudié en vue d'appuyer cette qualification. En effet, en guise de synonyme de «excision" le Commissaire du Gouvernement emploie l'expression «mutilation génitale féminine", mettantl'accent sur le caractère mutilateur de cette pratique et passant sous silence son aspect culture ${ }^{16}$. Le tribunal administratif conclue enfin que les filles de Mariama étaient réellement menacées d'excision en cas de retour en Guinée. En effet, contrairementà la CRR, il reconnaît l'existence de différentes sphères normatives (le droit étatique et les règles familiales) dans la société guinéenne, perméables les unes aux autres. Ainsi, le droit étatique guinéen a officiellement intégré la pratique de l'excision en ne prévoyant aucune répression contre elle. En dernier lieu, le tribunal récuse un raisonnement métonymique très largement utilisé dans la logiquejuridique. Il précise en effet que le fait que la Guinée ait signé et ratifié la Convention relative aux droits de l'enfant, la Convention pour l'élimination de toutes les formes de discrimination à l'égard des femmes et la Charte Africaine, ne signifie pas qu'elle applique ces accords. La ratification d'une convention (une partie de la mise en place de la protection des personnes) n'est pas équivalente à la protection effective de ces personnes (le tout).

\section{Analyse des concepts structurants du droit applicable aux demandeurs d'asile}

La mise en parallele des deux dispositifs des décisions conduit à énoncer la conclusion suivante: d'une part, Mariama et ses fillesn'ont pas de craintes fondées de persécutions et ne peuvent donc obtenir le statut de réfugiées, d'autre part, les enfants de Mariama risquent de subir un traitement inhumain et dégradant en cas de retour en Guinée et leur mère ne peut donc être expulsée en direction de la Guinée. Or, comment peut-onne pas avoir raison de craindre des persécutions si l'on est menacée de traitements inhumains et dégradants? Ces conclusions mettent en relief la spécificité du raisonnement juridique et ce en quoi il s'écarte de la logique formelle et du bons sens.

En droit, il est possible d'accepter deux décisions affirmant que des craintes de persécutions ne sont pas fondées mais qu'un risque de traitement inhumain et dégradant existe car ce raisonnement repose sur un concept structurant du droit, sur une métaphore devenue conventionnelle, celle de «branches» du droit. Ce trope, issu de la métaphore plus vaste de «l'arborisation» du droit, implique à une différenciation entre les différents domaines du droit qui partent tous d'un tronc commun. La grande division, celle qui distingue le droit public du droit privé, possède de nombreuses ramifications. Ainsi, dans le domaine du droit public, on trouvera unebranche «droit des réfugiés», une autre "droit administratif», elle-même ayant une ramification "contentieux administratif», etc. Cette métaphore est devenue un concept structurant du droit car les juristes ne la remarquent plus, l'acceptent comme une évidence ètne peuventl'éviter. Elle est ainsi un élément incontournable de la façon dont on pense en droit, et c'est effectivement en fonction de ces différentes branches qu'a été déterminé le cas de Mariama.

A chaque branche du droit correspond certaines «sources" de droit et certaines procédures. Ainsi, le statut de réfugié politique est déterminé en fonction dela notion de "persécution" qui se rattache à la Convention de Genève de 1951 et une procédure devant l'OFPRA et la CRR. En revanche, l'appréciation du pays vers lequel une personne est expulsée se fait en fonction de la notion de «torture et peine ou traitement inhumain et dégradant's issue de la Convention européenne de sauvegarde des droits de l'homme et des libertés fondamentales et selon les règles du contentieux administratif. Ce processus de différencia- 
tion permet donc d'affirmer, juridiquement, qu'une persécutionn'est pas une torture, qui n'est non plus équivalenteà un traitement inhumain et dégradant, etc. Il se crée donc une structure de pensée qui est rationnellement fausse, tout comme le sont les images issues de métaphores. En effet, rationnellement et humainement, $l$ 'excision est une pratiquedangereuse, humiliante, qui provoque de très fortes souffrances et la personne qui en est victime la perçoit à la fois comme une torture, un mauvais traitement, une persécution. Ce décalage dans lequel seplace la logique juridique est la source du manque de compréhension de nombreuses décisions de justice. En effet, la réaction naturelle d'une personne qui prend connaissance de l'histoire de Mariama est de se demander s'il est ou non dangereux pour elleetses enfants de retourner en Guinée. La réponse apportée par les deux dispositifs ici analysés ne peut être satisfaisante à cet égard.

Il convientcependantd'aller au-delà de cette première analyseet des' interroger sur les conséquences de cette différenciation. Ainsi, la notion de craintes de persécution est interprétée de façon plus restrictive que celle de traitement inhumain et dégradant. Le droit français peut ainsi s'affirmer respectueux des droits del'homme caril autorise une interprétation très large de la notion de risques encourus dans le pays de retour. Mais cette interprétation libérale n'intervient qu' une fois qu' une personne a faitl'objet d'un arrêté de reconduiteà la frontière, donc une fois qu'il est certain que, quelque soit le pays vers lequel elle sera expulsée, elle ne restera pas en France. En revanche, l'interprétation de la notion de persécution et les procédures devant 1'OFPRA et la CRR sont plus restrictives car la conséquence de la reconnaissance d'un risque de persécution estl'octroi du statut de réfugié et le droit de rester en France. La métaphore des «branches» du droit permet doncà la France de restreindre le droit de rester sur le territoire par la reconnaissance du statut de réfugié, mais elle masque cette restriction en mettanten lumièrele fait qu'ellen'expulse pas des personnes vers des pays où elles risqueraient de subir des traitements inhumains et dégradants. Ainsi, le droit applicable aux demandeurs d'asile est largement fonction de la politique d'immigration du pays.

Cet article s'est limité à l'analyse du langage utilisé par les juridictions. En conclusion, il convient cependant de mentionner le rôle joué par le langage, en amont, lors de l'elaboration de lois relatives au droit d'asile. En effet, lors des débats parlementaires ou dans les médias, les métaphores utilisées en matière de libre circulation des personnes mettent généralement l'accent sur l'idée de sécurité. Ainsi, une frontière devient un «verrou», l'Europe une «forteresse", etc. Or, par comparaison, il convient de remarquer que l'image fréquemment utilisée en matière de libre circulation des marchandises ou des capitaux est celle du "village planétaire" qui, de façon beaucoup plus positive, évoque la convivialité, l'échange, le partage. Ces tropes, sortes de messages subliminaux, sont importants car ils influencentl'adhésion de la population àl'égard desnouvelles loiset confortent ainsi l'un des concepts structurants métonymiques les plus important du droit français: «la loi est la volonté du peuple».

\section{Notes}

1. Trope "qui consiste dans un transfert de sens par substitution analogiquen (Définition du Petit Robert).

2. Trope upar lequel on exprime un concept au moyen d'un terme désignant un autre concept qui lui est uni par une relation nécessaire (la cause pour l'effet, le contenant pour le contenu, le signe pour la chose signifiée)" (Définition du Petit Robert).

3. Je suis consciente que la présentation sommaire et partielle des faits telle que je la livre ici correspond précisément à la démarche queje vais tenter de dénoncer dans le paragraphe suivant. Il s'agit d'une lecture selective des faits en fonction des elements juges importants parl'auteur selon ce qu'elle souhaite montrer. Cette contradiction est da au fait que cet article ne doit pas excéder une certaine longueur. J'encourage vivement les lectrices et lecteurs à se rapporter aux décisions citées afin de donner tout leur sens aux commentaires qui vont suivre.

4. Commission des Recours des Réfugiés, 1er mars 1996, requête $n^{\circ} 295574$.
5. Article 1er, paragraphe A: «Autermedela présente Convention, le terme "réfugié"

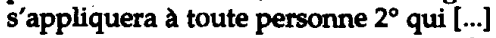
craignant avecraison d'être persécutée du fait de sa race, de sa religion, de sa nationalité, de sont appartenance à un certain groupe social ou de ses opinions politiques, se trouve hors du pays dont elle a la nationalité et qui ne peut ou, du fait de cette crainte, ne veutse réclamer dela protection de ce pays, ou qui, si ellen'a pas de nationalité et se trouve hors du pays dans lequel elle avait sa résidence habituelleàla suite de tels événements ne peut ou, en raison de ladite crainte, ne veut y retourner". Convention relative statut des réfugiés et des apatrides, 28 juillet 1951, (1954) 189 R.T.N.U. 137.

6. CRR, 1er mars 1996, requête $n^{\circ} 295574$.

7. Tribunal administratif de Lyon, $6 \mathrm{e}$ chambre, $n^{\circ}$ 9600127, 29 mai 1996.

8. Article 3: «Nul ne peut être soumis à des tortures ni à des peines ou traitement inhumains et dégradants" Convention européenne de sauvegarde des droits de l'homme et des libertés fondamentales, 4 novembre 1950 , S.T.E. 5, 213 R.T.N.U. 221.

9. A. L. Goodhart, Essay in Jurisprudence and the Common Law, Cambridge, The University Press, 1931, p. 8.

10. Cons. d'Etat, 2 décembre 1994, Mme Agyepong, (1994) A.J.D.A., p. 915.

11. Les décisions de justice françaises sont généralement courtes et leur motivation brève. Cette technique permet desuggérer qu'aucun douten'était possible quantà la décision à prendre, que celle-ci s'imposait.

12. LaCRR a considéré que epostérieurement aux faits invoqués pour justifier son départ de Guinée, la requérante a obtenu, sans alléguer qu'elle y auraitété contrainte par une nécessité impérieuse, la prorogation de son passeport le 8 février 1995 auprès del 'ambassade de Guinée a Paris; qu'en se plaçant ainsi sous la protection des autorités de son pays d'origine, elle ne peut plus être regardée comme étant au nombre des personnes visees par les stipulations précitées de la convention de Genève».

13. LaCRR a considéré que «il n'est pas établi que les membres de l'ethnie malinké proches de l'ancien président Sékou TOURE continueraient d'étre victimes de menaces volontairement tolérées par les autorités publiques".

14. LaCRR a pourtant déjà reconnu que, dans certains cas, l'excision pouvait être qualifiée de persécution des femmes volontairement tolérée par les pouvoirs publiques de certains Etats africains (CRR, 17 juillet 1991, Melle Aminata Diop, requête ${ }^{\circ} 164$. 078). 
15. D'autres facteurs peuvent expliquer quela parole de Mariaman'ait pu être entendue. Ainsi est-il précisé dans la décision de la CRR que le français n'etait pas la langue maternelle de Mariama. De plus, il peut exister des entraves institutionnelles à la libre expression d'une réfugiée. Par exemple, il peut être difficile de s'expliquer clairement et totalement sur des craintes intimes, devant plusieurs hommes, étrangers.

16. A l'inverse, certains auteurs emploieront l'expression "circoncision féminine» qui, faisant l'analogie avec la circoncision masculine, insiste sur l'aspect culturel decette pratique.Cependant, ellen'insiste pas sur lefait que, pratiquée sur une fille, elleaboutit à une véritable mutilation. $\square$

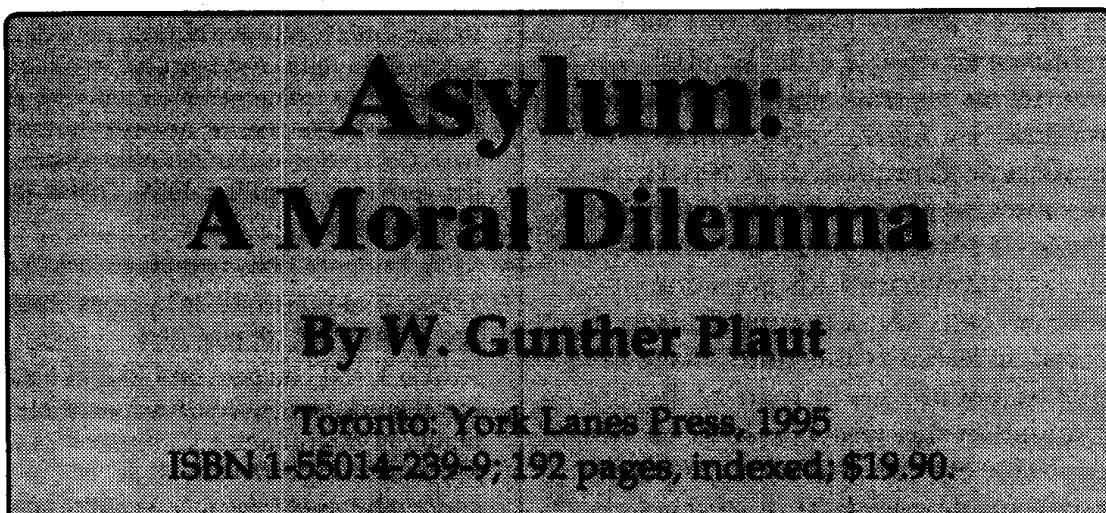

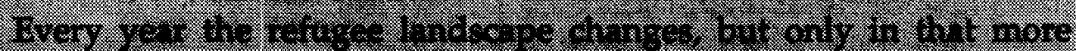

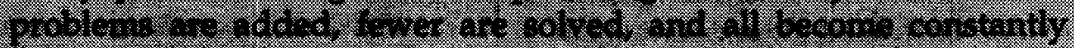

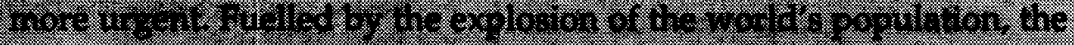

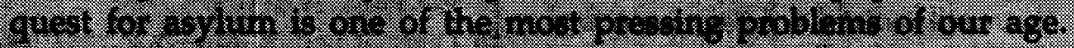

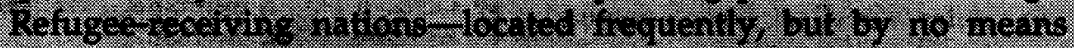

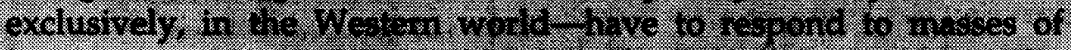

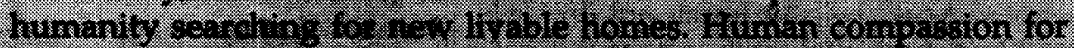

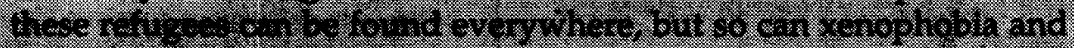

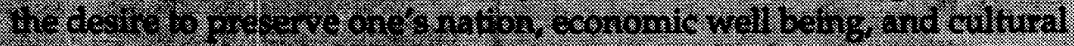

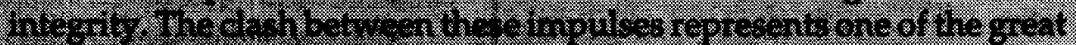

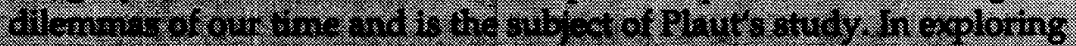

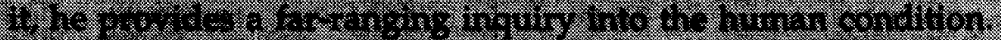

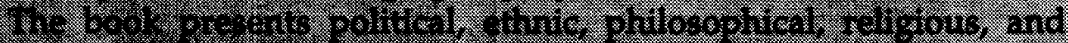

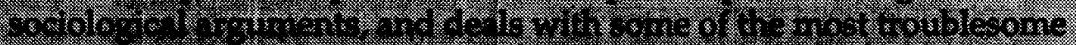

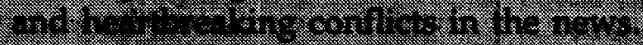

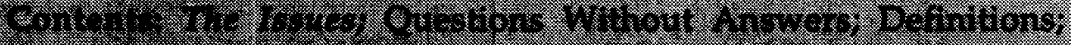

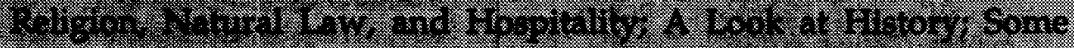

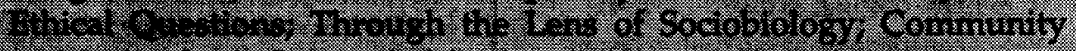

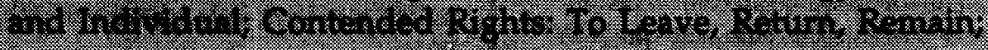

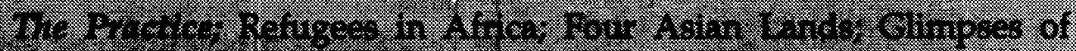

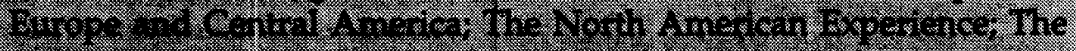

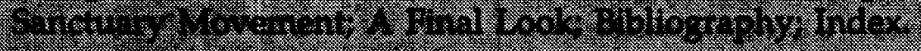

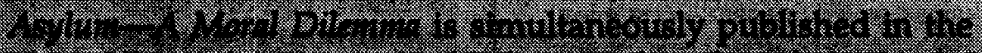

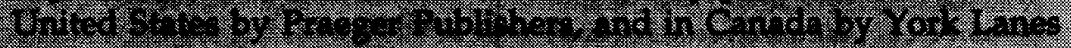
1.:5:

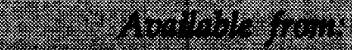

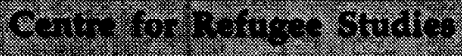

From Being

Uprooted to

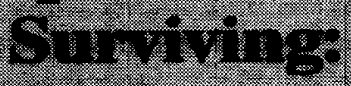

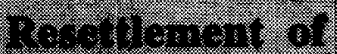

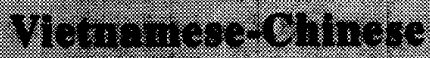

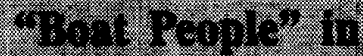

Montreal, 1000-1990

\section{By Lawrence Lam.}

Toronto: York Lancs Pros

ISBN 1-55014-296-8

200. por.8s: inderedi: $\$ 18.95$

The sager of the 'boar people' is a atrinticestory, a story of one of the lingest reftiger morements in te-

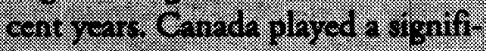
cant role in the resetiement of

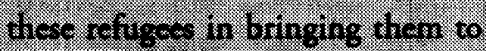
Canade where thoy could start ancer. Fron Bing Uproosed to Sur. viving by Professor Lam, is based on ethinozrephic data of a sample of Vhentinese-Chinese-eccopted for resertement in Montral in 1979 and 1980, who werte interviewed again in 1984-85 and in 1990 91. this book provides a longitudinal accoumt of their epperience of resertlencir in Cande. This expericnes her been marked by succes-

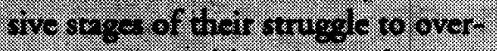
como strumuint batriess and to nogotiate a merningful niche in Canad:

Coneents: Prefree, The Boar People Phanomenon, ResettionientIssues and I Tespectives, The Viet-

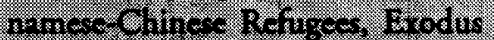
and T mondion. Reserdement Proc-

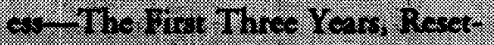
dement-f fornd the Firet Thee Vears. Conclusion.

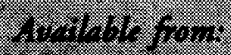

Crater tor Refubes Smitis

P.r. (416) 736-5837.

2. 